the lifetimes and intensities of positrons annihilating in polysyrene (to be reported ${ }^{10}$ ) will be summarized in support of the above discussion. We find three components in this glass. $I_{1}(\simeq 35 \%)$ is due to free positrons not trapped or in some short-lived bound state $(\simeq 20 \%)$ and $p$-Ps $(\simeq 15 \%)$. $I_{2}(\simeq 27 \%)$ is due to the annihilation of free positrons in regions of order due to nodules ${ }^{11}$ or spherically symmetric strain fields. ${ }^{12} I_{3}(\simeq 38 \%)$ is due to the annihilation of positrons in the amorphous regions by pickoff of $o-\mathrm{Ps}$. A plot of $\tau_{3}$ ( $\simeq 2 \mathrm{nsec}$ ) versus the temperature $T$ shows a linear relationship with positive slope, changing to a steeper positive slope at the glass transition. ${ }^{6}$ A plot of $\tau_{2}(\simeq 0.4 \mathrm{nsec})$ versus $T$ shows a linear relation with positive slope, similar to $\tau_{3}$ versus $T$, below the glass transition where $\tau_{2}$ changes abruptly from about 0.42 to $0.47 \mathrm{nsec}$. Thus the glass transition seems to consist of a combination of first-order and second-order transitions. The lifetime of the $\tau_{1}$ component changes abruptly from $0.13 \mathrm{nsec}$ in the glass to about $0.16 \mathrm{nsec}$ in the highly viscous liquid. While results for temperatures below the glass transition were easily fitted by our model, results for temperatures above the glass transition (highly viscous liquid) were difficult to fit, although the data fit three components better than two or four. Experiments to measure the temperature dependence of positron lifetimes and intensities in polyethylene and to continue the electric field experiments for polar molecules, such as Teflon, are planned.

The authors are grateful for the advice and assistance of I. K. MacKenzie. In particular his suggestion of a time-of-flight time calibration system is acknowledged. The help of C. Y. Leung in initiating this experiment is also acknowledged as is the technical assistance of R. Gingerich.

\footnotetext{
*Research supported by the National Research Council of Canada.

${ }^{1}$ P. C. Lichtenberger, J. R. Stevens, and T. D. Newton, Can. J. Phys. 50, 345 (1972).

${ }^{2}$ W. Brandt and I. Spirn, Phys. Rev. 142, 231 (1966).

${ }^{3}$ J. D. McGervey and V. F. Walters, Phys. Rev. B $\underline{2}$, 2421 (1970).

${ }^{4}$ J. R. Stevens and M. J. Edwards, J. Polymer Sci., Part C 30, 297 (1970).

${ }^{5}$ Pressure Chemical Company, 3419-25 Smallman Street, Pittsburgh, Pa. 15201.

${ }^{6}$ J. R. Stevens and A. C. Mao, J. Appl. Phys. 1ㅡ, 4273 (1970).

${ }^{7}$ W. Brandt and H. Feibus, Phys. Rev. 174, 454 (1968).

${ }^{8}$ B. Wunderlich and H. Baur, Advan. Polymer Sci. $\underline{7}$, 151 (1970).

${ }^{9}$ I. K. MacKenzie and B. T. A. McKee, Can. J. Phys. 44, 435 (1966).

${ }^{10}$ J. R. Stevens, A. C. Mao, and P. C. Lichtenberger, to be published.

${ }^{11}$ G. S. Y. Yeh and P. H. Geil, J. Macromol. Sci., Phys. 2, 235 (1967).

${ }^{12}$ J. R. Stevens, I. C. Bowell, and J. L. Hunt, to be published.
}

\title{
Close Similarity between Photoelectric Yield and Photoabsorption Spectra in the Soft-X-Ray Range
}

\author{
W. Gudat and C. Kunz \\ Deutsches Elektronen-Synchrotron, Hamburg, Germany
}

(Received 19 May 1972)

\begin{abstract}
Because of the similarity between photoelectric yield and photoabsorption spectra in the soft $x$-ray region, "yield spectroscopy" can be used as a successful method for investigating fine structure in the electronic excitation spectra of solids. The continuous spectrum of synchrotron radiation is especially appropriate for such measurements. We reinvestigated the structure of the $4 d$ excitations in $\mathrm{Pr}$ and the $2 p$ excitations of Si using a single crystal.
\end{abstract}

Using the continuous spectrum from a soft-xray tube, Lukirskii and co-workers ${ }^{1,2}$ established a close similarity between photoelectric yield and absorption spectra for core excitations in several materials, mainly alkali halides. There are, however, only a few yield measurements available which allow a comparison in the low-en- ergy region. Good agreement is found for the $L_{2,3}$ spectrum $^{3}$ of $\mathrm{Na}$ in $\mathrm{NaCl}$ around $30 \mathrm{eV}$. At even lower energies the $3 p$ excitations of $\mathrm{K}$ in the potassium halides ${ }^{4,5}$ around $20 \mathrm{eV}$ can be identified in both absorption $\left(\epsilon_{2}\right)$ and yield spectra; the actual shape, however, of the structure is quite distorted in the yield spectra. The valence-band 
spectra at the onset of transitions appear to be different in yield and absorption. ${ }^{405}$ Few yield investigations have been performed on clean metal and semiconductor surfaces. An unpublished yield measurement ${ }^{6}$ on an oxide-free Al surface agrees well with the known $L_{2,3}$ absorption spectrum. This indicates that the similarity between the two types of spectra is not restricted to insulators.

Synchrotron radiation ${ }^{7}$ with its intense continuous spectrum covering the whole ultraviolet and soft-x-ray region is especially suited for "yield spectroscopy." In order to further explore the possibilities of this method we have performed photoelectric yield measurements with a variety of insulators, semiconductors, and metals. For LiF, e.g., we were able to confirm the published results $^{2}$ and found excellent agreement in all the details of the structure with more recent absorption measurements ${ }^{8,9}$ in the $60-150 \mathrm{eV}$ range. We were able to reproduce the absorption spectrum $^{10}$ of CsI between 50 and $170 \mathrm{eV}$ (with the Cs and I $N_{4,5}$ structures) and saw the increase in the absorption coefficient ${ }^{11}$ of CdSe (below $110 \mathrm{eV}$ ) and of $\mathrm{PbS}$ (below $80 \mathrm{eV}$ ). We similarly found the rapid increase in the absorption ${ }^{12}$ of $\mathrm{Ag}$ below $120 \mathrm{eV}$ together with some fine structure around $70 \mathrm{eV}$. Measurements on $\mathrm{Ce}, \mathrm{Pr}$, and a single crystal of Si will be discussed at the end of this paper. The present investigations were of an exploratory nature and will be followed by detailed measurements under ultrahigh-vacuum conditions at a later stage. All these results prove that in the extreme uv region yield spectroscopy is a powerful tool for the investigation of absorption structure having all the advantages of using bulk samples, e.g., single crystals.

Although we are aware that the general theory of photoemission is complicated, and that especially the processes involved during the migration of electrons to the surface are not very well understood, the following simplified considerations appear to give a reasonable explanation of the yield spectra. After the primary absorption of a photon, three processes mainly contribute to the photoelectric yield: (1) direct excitation into the conduction band, (2) Auger emission of electrons, and (3) decay of excitons into one-electron excitations.

The fast electrons originating from these processes undergo inelastic electron-electron scattering with the probability of multiplication, the multiplication factor $\eta(\epsilon)$ being a monotonic increasing function of the electron energy $\epsilon$. The main contribution to the yield should come from electrons with energies a few electron volts above the vacuum level because in the spectral region, which interests us, the mean free path for inelastic scattering increases with decreasing energy, whereas the escape probability through the surface barrier increases from the vacuum level to higher energies. This should result in an effective escape depth $D$ determined by the mean free path of these electrons. $D$ is on the order of $30 \AA$ for metals (see, e.g., Kanter ${ }^{13}$ ) and several times larger for insulators in the region where electron-electron scattering is impossible. $D$ is small compared to the penetration depth of light in our region of interest; as a result, the total yield $Y$, defined as the number of emitted electrons divided by the number of incident photons (near normal incidence the reflectivity is negligibly small), is given by

$$
Y \propto \mu(E) D\left[p_{1} \eta\left(\epsilon_{1}\right)+p_{2} \eta\left(\epsilon_{2}\right)+p_{3} \eta\left(\epsilon_{3}\right)\right],
$$

$\mu(E)$ being the absorption coefficient of the photons; $p_{1}, p_{2}$, and $p_{3}$ being the relative contributions of the processes (1) to (3); and $E$ being the photon energy. Apart from the $\epsilon$-dependent bracket, $Y$ is proportional to $\mu(E)$. Since the electron energies $\epsilon_{i}$ are determined by the photon energy $E$, the bracket term will become a monotonic increasing function of $E$. We presume that the function will be structureless due to the averaging over a series of statistical scattering processes which are involved in the determination of $\eta(\epsilon)$. Without going into the details of Eq. (1) for insulators, semiconductors, and metals we would like to mention two points: (a) As has been noted before by Lukirskii et al., ${ }^{2}$ the Auger part of Eq. (1) (apart from the region where it competes with excitonic decay) should be a true replica of $\mu(E)$. (b) Core excitons which predominantly decay into one-electron excitations are by no means suppressed in the yield spectra.

Our experiments were performed by making use of synchrotron radiation emitted by the 7.5GeV electron accelerator DESY. The continuum was monochromatized by a special monochromator, ${ }^{14}$ giving a light beam virtually free from higher-order radiation at a fixed exit slit in the energy region 35 to $300 \mathrm{eV}$. The resolution $E /$ $\Delta E$ ranges from 400 to 650 . Behind the exit slit the light was reflected at a grazing angle of $4^{\circ}$ by a gold-coated mirror which, at the same time, was the cathode of an open multiplier (Bendix). The signal from this multiplier served as the reference for the yield measurements. The spec- 


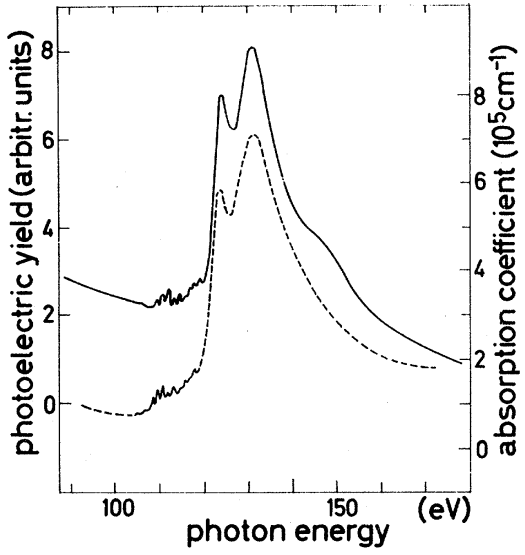

FIG. 1. Comparison of photoelectric yield (solid curve) and absorption (dashed curve) spectra for Pr.

trum, as measured by this detector, is smooth with the exception of small fine structure at the Au $N_{6,7}$ edges around $85 \mathrm{eV}$ originating from the gold coating of all the optical components and a pronounced structure at the carbon $K$ edge at 280 $\mathrm{eV}$ originating from hydrocarbon contamination on the optical surfaces. The reflected light beam hits the samples at normal incidence. The emitted electrons are accelerated onto the cathode of an open multiplier (Johnston). The signal of this multiplier is electronically divided by the reference signal. The spectra obtained in this way can differ from the actual yield by a factor varying smoothly with photon energy. We call the spectra thus measured "yield spectra."

Figures 1 and 2 show the yield and the absorption spectra ${ }^{15-17}$ of $\mathrm{Pr}$ in the region of $4 d$ transitions. Since the samples were not prepared in situ they will be covered by some layer of oxide. For $\mathrm{Pr}$ it has been shown before that the absorption spectra of oxide and metal agree with each other. The yield and absorption spectra show very good agreement. (The broad bump at 150 $\mathrm{eV}$ also shows up in the absorption spectrum of Ref. 16.) In the yield spectrum the underlying continuum of transitions from higher shells is more pronounced, compared to the fine structure, than in the absorption spectrum. Similar behavior was observed with most of the other materials mentioned above and should be explainable from a detailed consideration of Auger and other excitations for the transitions from different shells for each individual material according to Eq. (1). An equally good agreement between the yield and the absorption spectrum was obtained from measurements on $\mathrm{Ce}$.

More details of the fine structure are observed

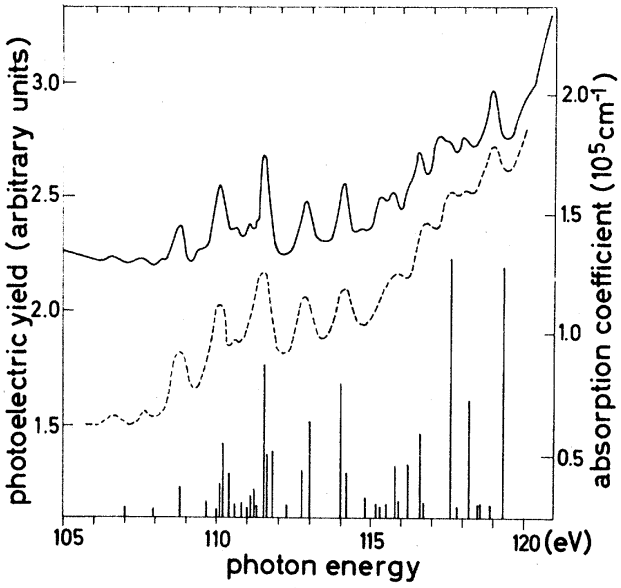

FIG. 2. Energy region of fine structure, yield (solid curve) and absorption (dashed curve) for Pr. The vertical lines are the result of an atomic calculation (Ref. 19); the length of the lines represents the oscillator strength.

in the yield spectra when compared to the absorption spectra primarily as a result of a better signal-to-noise ratio. This advantage is inherent in yield spectroscopy. A theoretical explanation of the structures in the rare-earth metals was achieved by Dehmer et al. ${ }^{18}$ and Sugar $^{19}$ on the basis of atomic calculations. Because of exchange interaction the $4 d^{9} 4 f^{N+1}$ final-state configuration splits into a series of widely spread sublevels. The numerical calculations performed for $\mathrm{Ce}$ and $\mathrm{Pr}$ show good agreement, also for the additional lines not found in the absorption spectra, both in position and strength of the lines (see, e.g., Fig. 2).

In addition we performed measurements on a

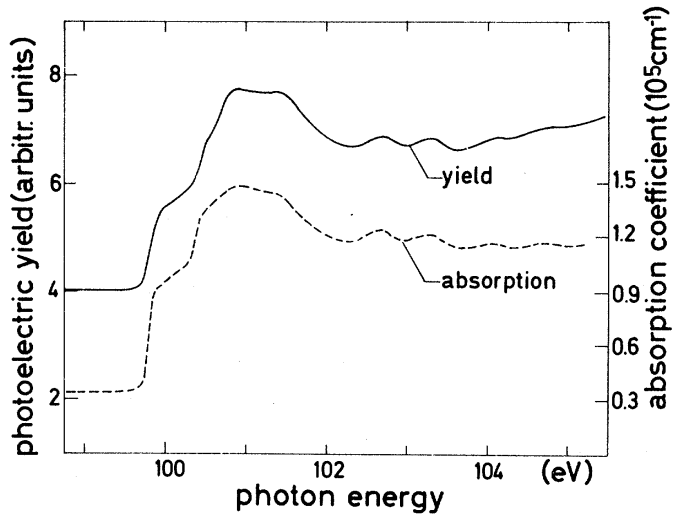

FIG. 3. Comparison of the photoelectric yield measured on a cleaved Si single crystal surface (solid curve) and the absorption coefficient (dashed curve) of a polycrystalline Si film, after Brown and Rustgi (Ref. 20). 
Si single crystal cleaved in air immediately before mounting. The result is shown in Fig. 3 and is compared with the absorption measurements performed by Brown and Rustgi ${ }^{20}$ on a polycrystalline sample. Small differences could be mainly attributed to a lower resolution in our case. The main features, however, are present in both spectra. The rise of the yield at the high-energy end of the spectrum is due to the first peak in the spectrum ${ }^{21}$ of $\mathrm{SiO}_{2}$ at $106 \mathrm{eV}$ since our sample was not completely free from oxide. (This was even more pronounced when using a polished single crystal.) When measurements under ultrahigh-vacuum conditions become possible, the investigation of single crystals by yield spectroscopy will certainly be of primary interest.

We would like to thank M. Skibowski for his critical reading of the manuscript.

\footnotetext{
${ }^{1}$ A. P. Lukirskii and I. A. Brytov, Fiz. Tverd. Tela $\underline{6}$, 43 (1964) [Sov. Phys. Solid State $\underline{6}, 32$ (1964)]; A. P. Lukirskii and T. M. Zimkina, Izv. Akad. Nauk SSSR, Ser. Fiz. 28, 765 (1964).

${ }^{2}$ A. P. Lukirskii, O. A. Ershov, T. M. Zimkina, and E. P. Savinov, Fiz. Tverd. Tela $\underline{8}, 1787$ (1966) [Sov. Phys. Solid State 8,1422 (1966)].

${ }^{3}$ R. Haensel, G. Keitel, G. Peters, P. Schreiber, B. Sonntag, and C. Kunz, Phys. Rev. Lett. 23, 530 (1969).

${ }^{4}$ D. Blechschmidt, M. Skibowski, and W. Steinmann, Opt. Commun. 1, 275 (1970).

${ }^{5}$ T. Sasaki, Y. Iguchi, H. Sugawara, S. Sato, T. Nasu,
}

A. Ejiri, S. Onari, K. Kojima, and T. Oya, J. Phys. Soc. Jap. 30, 581 (1971).

${ }^{6} \mathrm{G}$. Peters, Diplomarbeit, University of Hamburg, 1969 (unpublished).

${ }^{7}$ R. Haensel and C. Kunz, Z. Angew. Phys. 23, 276 (1967); R. P. Godwin, in Springer Tracts in Modern Physics, edited by G. Höhler (Springer, Berlin, 1969), Vol. 51.

${ }^{8}$ R. Haensel, C. Kunz, and B. Sonntag, Phys. Rev. Lett. 20, 262 (1968).

${ }^{9}$ F. C. Brown, C. Gähwiller, A. B. Kunz, and N. O. Lipari, Phys. Rev. Lett. 25, 927 (1970).

${ }^{10}$ M. Cardona, R. Haensel, D. W. Lynch, and B. Sonntag, Phys. Rev. B 2 , 1117 (1970).

${ }^{11}$ M. Cardona and R. Haensel, Phys. Rev. B 1,2605 (1970).

${ }^{12}$ R. Haensel, C. Kunz, T. Sasaki, and B. Sonntag, Appl. Opt. 7, 301 (1968).

${ }^{13}$ H. Kanter, Phys. Rev. B 1,522 (1970).

${ }^{14}$ H. Dietrich and C. Kunz, Rev. Sci. Instrum. $\underline{43}, 434$ (1972).

${ }^{15}$ V. A. Fomichev, T. M. Zimkina, S. A. Gribovskii, and I. I. Zhukova, Fiz. Tverd. Tela 9, 1490 (1967)

[Sov. Phys. Solid State 9 , 1163 (1967)].

${ }^{16}$ T. M. Zimkina, V. A. Fomichev, S. A. Gribovskii, and I. I. Zhukova, Fiz. Tverd. Tela 9, 1447 (1967) [Sov. Phys. Solid State 9, 1128 (1967)].

${ }^{17}$ R. Haensel, P. Rabe, and B. Sonntag, Solid State Commun. $\underline{8}, 1845$ (1970).

${ }^{18}$ J. L. Dehmer, A. F. Starace, U. Fano, J. Sugar, and J. W. Cooper, Phys. Rev. Lett. 26, 1521 (1971).

${ }^{19} \mathrm{~J}$. Sugar, Phys. Rev. B 5,1785 (1972).

${ }^{20}$ F. C. Brown and O. P. Rustgi, Phys. Rev, Lett. $\underline{28}$, 497 (1972).

${ }^{21}$ O. A. Ershov and A. P. Lukirskii, Fiz. Tverd. Tela 8, 2137 (1966) [Sov. Phys. Solid State $\underline{8}, 1699$ (1967)].

\title{
Magnetic Field Effect on Plasma-Wave Dispersion in a Dielectric Layer
}

\author{
R. E. De Wames and W. F. Hall \\ North American Rockwell Science Center, Thousand Oaks, California 91360
}

(Received 3 April 1972)

\begin{abstract}
We present dispersion curves and field amplitudes for a plasma layer in a magnetic field. Various effects such as the nonreciprocal character of the dispersion, the pileup of bulk states at $\omega_{\infty}$, and the existence of surface states above $\omega_{\infty}$ should be observable in optical experiments.
\end{abstract}

The recent measurements of the surface plasma dispersion relation in InSb by Marschall, Fischer, and Queisser, ${ }^{1}$ are in good agreement with predictions derived by treating the semiconductor as a dielectric half-space. ${ }^{2}$ However, the dispersion relation in this case is a single, structureless curve asymptotic at small frequency to the light-line $(\omega=k c)$ and at large $\omega$ to the surface plasma frequency $\left.\omega_{s}=\omega_{p}[\kappa / \kappa+1)\right]^{1 / 2}, \kappa$ being the high-frequency dielectric constant of the lattice. A more exacting test of the dielectric model can be made if the system is perturbed by the introduction of a magnetic field permeating the sample, and a finite thickness is considered for the dielectric.

The effect of a magnetic field on the optical constants deduced from bulk properties has been discussed by Palik and Wright. ${ }^{3}$ The modes dis- 